\title{
Development of SSR Markers Associated with Polima CMS Fertility Restorer in Brassica napus L. and Its Application
}

Tonghua Wang, Bao Li, Yiming Guo, Yaoting Zhang, Xinhong Liu, Lianyi Fan, Liang Qu, Lichao Deng, Mei Lið Hunan Crop Research Institute, Hunan Hybrid Rapeseed Engineering and Technology Research Center, Changsha, 410125, P.R. China

$\square$ Corresponding author email: Limei1230@126.com

Molecular Plant Breeding, 2021, Vol.12, No.17 doi: $10.5376 / \mathrm{mpb} .2021 .12 .0017$

Received: 19 May, 2021

Accepted: 24 May, 2021

Published: 03 Jun., 2021

Copyright $@ 2021$ Wang et al., This article was first published in Molecular Plant Breeding in Chinese, and here was authorized to translate and publish the paper in English under the terms of Creative Commons Attribution License, which permits unrestricted use, distribution, and reproduction in any medium, provided the original work is properly cited.

Preferred citation for this article:

Wang T.H., Li B., Guo Y.M., Zhang Y.T., Liu X.H., Fan L.Y., Qu L., Deng L.C., and Li M., 2021, Development of SSR markers associated with Polima CMS fertility restorer in Brassica napus L. and its application, Molecular Plant Breeding, 12(17): 1-7 (doi: 10.5376/mpb.2021.12.0017)

\begin{abstract}
The Polima system of cytoplasmic male sterility (Pol CMS) was widely used for hybrid seed production, but with high probability of occurrence of maternal false hybrid due to the fertility of Polima CMS (Pol CMS) line greatly affected by the ambient temperature, therefore, the detection of maternal false hybrids is the key to purity identification. In present study, the SSR markers were designed based on the flanking sequences of Pol CMS restorer gene $R f p$, and application of SSR markers in hybrid seed purity test of rapeseed. The main results were as follows: the sequences of $R f p$ were subjected to basic local alignment search tool queries against the Brassica rapa genome to determine chromosome positions, and 32 SSR markers were developed based on the upstream and downstream sequences of Rfp. Among the 32 SSR markers, one codominant marker was polymorphic between Pol CMS sterile and restorer line, the PCR product length was $162 \mathrm{bp}$ in Pol CMS sterile lines, and $184 \mathrm{bp}$ in restorer lines. Based on the PCR sequence, two insert fragments (15 bp and $7 \mathrm{bp}$ ) were identified in Pol CMS restorer lines. The SSR marker co-segregated with plant fertility of individuals in the "fengyou 737" $F_{2}$ population. The purity of "fengyou737", "fengyou320" and "fengyou958" were identified by means of PCR-SSR markers and field-planting, and the results of two ways were highly consistent, indicated that the codominant SSR marker tightly linked to Rfp, and the marker can detect paternal and maternal false hybrids. The present findings will provide technical assistance for hybrid seed purity test and genetic diversity analysis in rapeseed.
\end{abstract}

Keywords Brassica napus L.; Pol CMS; Restorer gene; SSR

Polima cytoplasmic male sterility (Pol CMS) is the first cytoplasmic male sterility type of Brassica napus with production value. It is also the sterile source of the most widely used hybrid pollination control system in rapeseed in China ( $\mathrm{Fu}, 1995)$. The sterility of the male sterile lines in this system is controlled by the interaction between the mitochondrial male sterile genes and the nuclear genome, and the fertility restorer gene (Rfp) is located in the nuclear genome (Yang et al., 1996). The fertility of this type of sterile line is easily affected by ambient temperature, causing the occurrence of self-pollination by its own pollen in the process of large area hybrid seed production, which leads to the production of the maternal pseudo hybrid. Because the female pseudo hybrid is sterile, it not only affects the consistency of varieties, but also seriously affects the production advantage of varieties. Therefore, strict identification of seed purity and quality must be carried out before the application and production of rapeseed hybrids (Jean et al., 1997; Natasa et al., 2006; Natasa et al., 2010; Zhao et al., 2010). At present, the laboratory identification of rapeseed purity quality mainly includes three categories: isozyme electrophoresis, gliadin electrophoresis and molecular marker techniques. Among them, isozyme electrophoresis and gliadin electrophoresis have the advantages of simple technique, rapid identification and low cost, however, the polymorphism of isozyme and storage protein was relatively low, for some hybrid combinations, it is difficult to distinguish their parents from their hybrids, so the practical application of this technique is greatly limited. Molecular markers based on genomic DNA sequences, especially SSR markers developed in the early 1990s (Wang et al., 2010; Zhang et al., 2013). Compared with other molecular markers, this type of marker is relatively simple in operation and low in cost. Therefore, it has become the preferred type of molecular marker in the identification of purity and authenticity of crop varieties (He et al., 2009; Song et al., 2013; Wang et al., 2014; Zhu et al., 2015; Wang et al., 2016). However, with the narrowing of genetic diversity 
of breeding resources, the workload of SSR marker screening has been increasing. At the same time, breeders often start parental reproduction and cross production when the phenotypic traits are basically the same in the selection of hybrid parents. However, there is still separation of genetic information at the molecular level, which makes it difficult to ensure the accuracy of the purity identification of hybrid lines (Wang et al., 2009; Zhan et al., 2015; Li et al., 2016).

The results showed that the mono-dominant gene $R f p$ was responsible for the fertility recovery of Pol CMS of $B$. napus (Yang et al., 1996). In recent years, researchers have done a lot of genetic mapping of $R f p$, and developed a series of molecular markers linked to the fertility restorer genes (Liu et al., 2007; Yang et al., 2008). Rfp gene has been located in the N9 or N18 linkage group respectively. Until 2014, $R f p$ was mapped to the N9 linkage group of B. napus, and was restricted to the $29.2 \mathrm{k}$ region of A9 in B. rapa by molecular marker analysis. This region contains $7 O R F S$, of which $O R F 2$ is most likely involved in fertility recovery (Liu et al., 2012; Liu, 2012). The researcher then transferred the ORF2 into sterile lines of B. napus, the results showed that $O R F 2$ had the function of fertility recovery (Chen et al., 2014). Further sequence analysis showed that the coding region of $O R F 2$ was 1953 bp long (Liu et al., 2016). Meanwhile, the genome sequencing information of $B$. rapa. and B. napus was published in 2011 and 2014, respectively, which also provided a useful reference platform for the development of molecular markers.

In light of the actual demand for purity identification of Pol CMS hybrids, the sequence information of $R f p$ candidate gene region was used in this study to perform BLAST analysis on target sequences in B. napus genome and B. rapa genome to determine the position and sequence of the $P P R$ gene related to fertility restoration. And then, SSR markers were developed in the nearby region and used in the purity identification study of Pol CMS hybrids. It was expected to provide a more accurate and efficient detection method for the purity identification of rapeseed hybrids.

\section{Results and Analysis}

\subsection{Development of fertility restorer gene-linked SSR Markers}

Based on the 1953 bp sequence of ORF2 published by Liu et al. (2016), a highly homologous region (99\% homologous) was obtained on the A9 chromosome of B. rapa. A total of 32 SSR loci were obtained in the upper and lower $15 \mathrm{~K}$ region of this region, and 32 pairs of SSR primers were designed and synthesized online. The 32 pairs of primers were used for PCR amplification of genomic DNA of 10 tested parental materials, and one pair of SSR primer RfpSSR19 (F: AGTTAGCTCCTCTGTGGTTTGC; R: CTACAGCTCGATTAGGGATCGT), and its amplification site was located at NC_024 803.1:34 437588 to 34437748 of B. napus genome. It showed significant polymorphism in tested 4 male sterile lines and 6 restorer lines (Figure 1), which was a co-dominant marker of sterile lines and restorer lines. Sequencing results of the specific fragments showed that the size of the amplified fragments was $162 \mathrm{bp}$ in the sterile line and $184 \mathrm{bp}$ in the restorer line. Compared with the sterile lines, there were one $15 \mathrm{bp}$ and one $7 \mathrm{bp}$ inserts in the amplified fragments of the restorer lines (Figure 2). The amplified product of RfpSSR19 was compared in B. napus, and the marker was located in the region from -3962 bp to -3 $801 \mathrm{bp}$ upstream of the initiation site of $R f p$ translation, indicating that the obtained marker RfpSSR19 was closely linked to the $R f p$ gene.

\subsection{Field location test of single plant of $F_{2}$ segregating population}

At the blooming stage, the $\mathrm{F}_{2}$ segregating population of the selfed progeny of the pol CMS hybrid Fengyou 737 was tested for fertility, and the SSR marker RfpSSR19 was used to analyze the genotypes of 192 randomly selected individual plants. The results showed that the single plant fertility of $184 \mathrm{bp}$ fragment in the amplified products was normal, and the single plant fertility of $162 \mathrm{bp}$ fragment was sterile, and the PCR amplification band pattern was co-segregated with the single plant fertility (Figure 3). 
Molecular Plant Breeding 2021, Vol.12, No.17, 1-7

$\mathrm{http}: / /$ genbreedpublisher.com/index.php/mpb

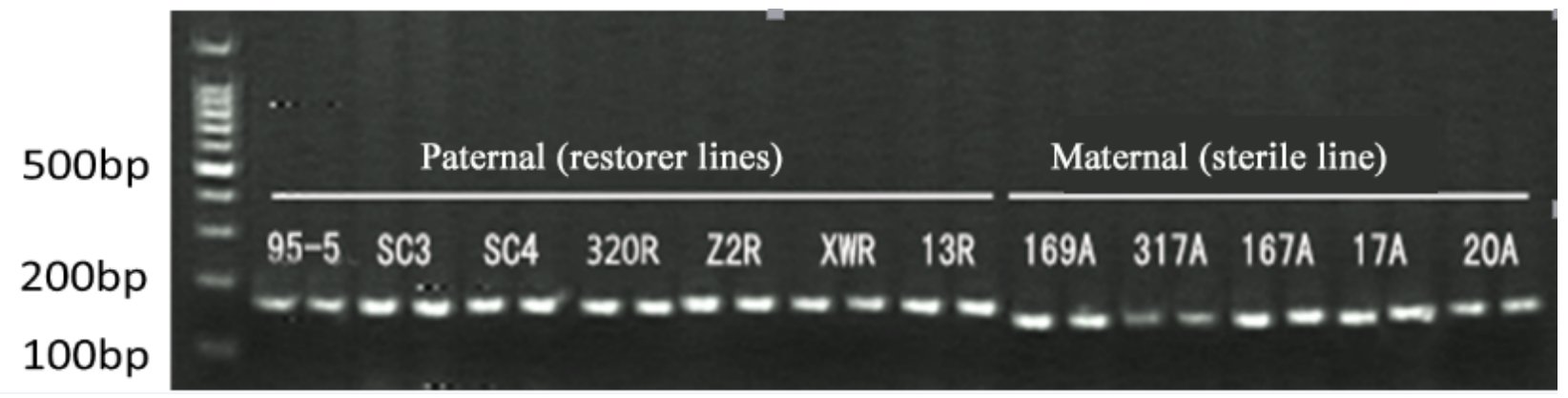

Figure 1 Banding patterns of the 12 parents of hybrids amplified by RfpSSR19

\begin{tabular}{|c|c|c|}
\hline $17 \mathrm{~A} . \mathrm{seq}$ & TAGTTAGCTCCTCTGTGGTTTGCCCGGTGCTCAAGAAACGATCTATTCCT & 50 \\
\hline 20A. seq & TAGTTAGCTCCTCTGTGGTTTGCCCGGTGCTCAAGAAACGATCTATTCCT & 50 \\
\hline sc3. seq & TAGTTAGCTCCTCTGTGGTTTGCCCAGTGCTCAAGAAACGATCTATTCCT & 50 \\
\hline SC4. seq & TAGTTAGCTCCTCTGTGGTTTGCCCAGTGCTCAAGAAACGATCTATTCCT & 50 \\
\hline $17 \mathrm{~A} . \mathrm{seq}$ & TTTT....... CTCGTCTTCGTTCGTCAGTTCGAGCTTCTTCATCTTCTT & 93 \\
\hline 20A. seq & TTTT....... CTCGTCTTCGTTCGTCAGTTCGAGCTTCTTCATCTTCTT & 93 \\
\hline sc3. seq & TTTTTTTCTTTCTCGTCTTCGTTCGTCAGTTAGAGCTTCTTCATCTTCTT & 100 \\
\hline SC4. seq & TтTтTт八TTTCTCGTCTTCGTTCGTCAGTTAGAGCTTCTTCATCTTCTT & 100 \\
\hline 17A. seq & стTстTCTCGCTT.................. & 128 \\
\hline 20A. seq & 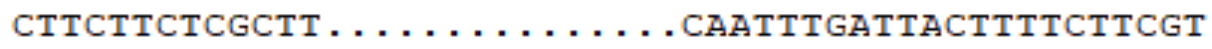 & 128 \\
\hline sc3. seq & СтTCTTCTCGCTTAACССTTATTTCTCTCAATTTGATTACTTTTCTTCGA & 150 \\
\hline sc4. seq & СTTСTTCTCGCTTAACССTTATTTCTCTCAATTTGATTACTTTTCTTCGA & 150 \\
\hline 17A. seq & CAAGGTATGTTTACGATCCCTAATCGAGCTGTAG & 162 \\
\hline 20A. seq & CAAGGTATGTTTACGATCCCTAATCGAGCTGTAG & 162 \\
\hline sc3. seq & CAAGGTACGTTTACGATCCCTAATCGAGCTGTAG & 184 \\
\hline SC4. seq & CAAGGTACGTTTACGATCCCTAATCGAGCTGTAG & 184 \\
\hline
\end{tabular}

Figure 2 Sequences comparison amplified by primers RfpSSR19 between two pol CMS lines and two restore lines

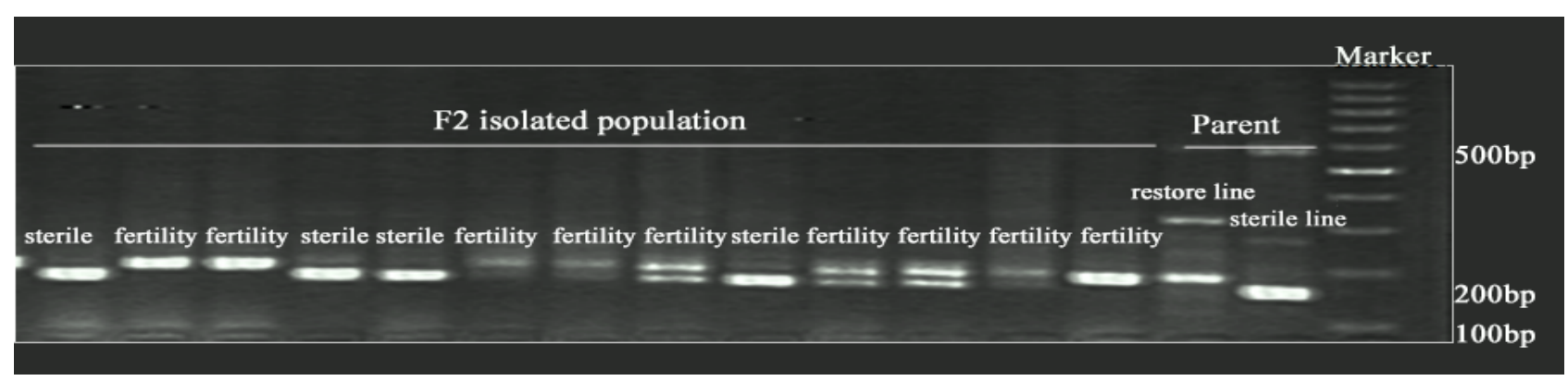

Figure 3 PCR amplification results and fertility performance of RpfSSR19 primer in pol CMS Fengyou $737 \mathrm{~F}_{2}$ segregating population

Note: 100 bp DNA Ladder

\subsection{Comparison of purity identification results of tested hybrid samples}

The selected SSR primers were used for PCR amplification of the genomic DNA of the seedlings of the tested hybrid varieties Fengyou 737, Fengyou 320 and Fengyou 958, and clear and stable amplification bands were obtained (PCR amplification results of Fengyou 737 hybrid samples) (Figure 4), and the reliable SSR purity identification results were obtained. As can be seen from the purity identification results of field planting (Table 1), the obtained results of SSR marker identification and field planting identification are highly consistent, and the 
difference of identification results of 9 tested samples is less than 4\%, indicating that marker RpfSSR19 is reliable for purity identification results of test materials used in this study.

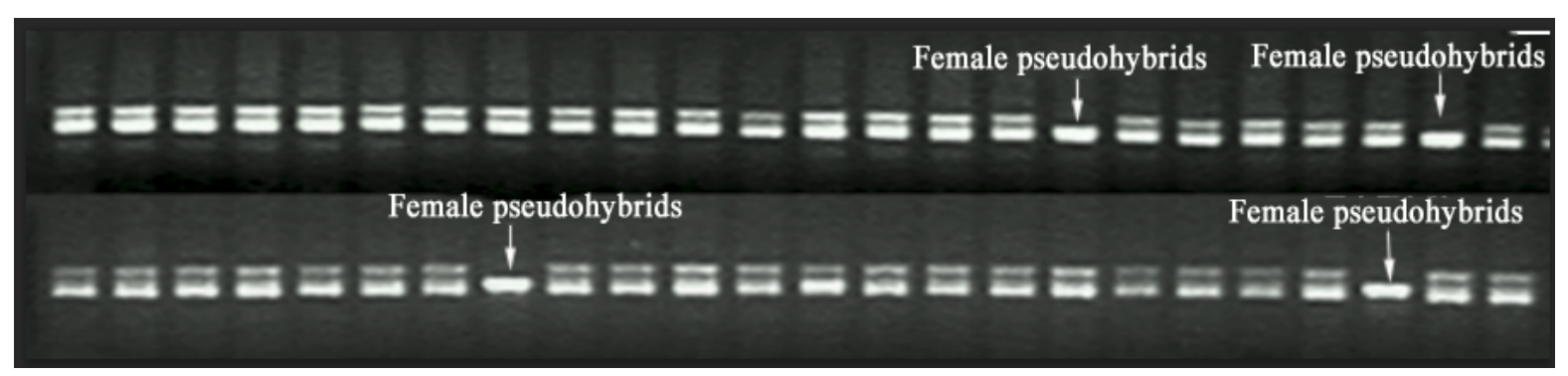

Figure 4 Amplification patterns of SSR-PCR in Fengyou 737 hybrids purity identification

Table 1 Purity of field planting identification and SSR identification of hybrid samples

\begin{tabular}{llll}
\hline Cultivars & No. & SSR purity $(\%)$ & Field purity $(\%)$ \\
\hline \multirow{3}{*}{ Fengyou 737} & $737-1$ & 89.5 & 91.6 \\
& $737-2$ & 92.3 & 91.5 \\
& $737-3$ & 87.9 & 90.2 \\
Fengyou 320 & $320-1$ & 89.3 & 92.2 \\
& $320-2$ & 88.8 & 89.1 \\
& $320-3$ & 87.9 & 90.8 \\
Fengyou 958 & $958-1$ & 92.1 & 90.5 \\
& $958-2$ & 90.3 & 93.6 \\
\hline
\end{tabular}

\section{Discussion}

The temperature sensitivity of male sterile lines is a problem in Pol CMS hybrid seed production system. For a long time, the monitoring of seed purity and quality, especially the accurate identification of female pseudo hybrids in hybrid lines, has been an indispensable link in rapeseed hybrid production (Zeng et al., 2009). In this study, a specific SSR marker closely linked to the $R f p$ gene was developed based on the actual requirements for purity identification of Pol CMS hybrids in rapeseed. The production and application tests showed that the marker could be used for purity and quality identification of different Pol CMS hybrids, and the identification results were highly consistent with the field fertility identification. The present study solves the problem that SSR markers for the purity of rapeseed hybrids need to be screened from genome-wide databases for the paternal and maternal lines of hybrid crossings (Mei et al., 2006; Tang et al., 2007). Therefore, a large number of preliminary works on the synthesis and screening of primer sequences is reduced, lowering the cost and early investment of identification, which is conducive to the popularization and application of the technology. At the same time, it overcomes the technical difficulty that common SSR markers can only be used to detect the genotypes of paternal and maternal lines of specific rapeseed hybrid crossings, which are greatly affected by biological and mechanical hybrid pseudohybrids, resulting in a certain deviation between identification results and field fertility identification.

At present, molecular markers based on PCR amplification, especially SSR markers, are abundant in genetic information due to their large number, high polymorphism, stable results and simple operation (Liu and Li, 2007; Chen et al., 2009), has become the preferred method for indoor purity identification of rapeseed hybrids. In this study, based on the advantages of common SSR markers, a specific SSR marker located in the -3962 bp to -3801 bp region upstream of the translation initiation site of $R f p$ gene was developed. Because it is difficult to recombine with the restorer gene, this marker can be used to identify the fertility of individual plants in the progeny of Pol CMS. Therefore, the results of purity identification by using it are more consistent with the individual fertility of field planting identification, and it has more practical significance in the purity identification of hybrids. At the same time, this marker is a co-dominant marker, which can effectively determine the pseudohybrid types of male and female parents in the hybrid samples, and can be used to determine the hybrid seeds of Pol CMS parents. The 
results obtained in this study can provide a new technique and method for the department of seed management and law enforcement.

The molecular marker identification purity of the 9 samples tested in this study was generally lower than that of field planting, but the differences were less than $4 \%$. It is speculated that it may be due to the stronger growth competitive advantage of hybrids in the process of field planting identification, which is easier to survive as a plant than its parents, leading to a higher results of fertility identification at flowering stage. In addition, the polymorphism of the markers obtained in this study was only $22 \mathrm{bp}$ difference between sterile and restorer lines, which required a long time to separate by agarose gel electrophoresis. Therefore, markers with more obvious differences of fragment size could be developed by further primer design to improve the efficiency of purity identification.

\section{Materials and Methods}

\subsection{Experimental materials}

The test materials: 12 parents of Pol CMS hybrid of Brassica napus, including 5 sterile lines and 7 Pol-CMS restorer lines (Table 2). Three samples of Fengyou 737, Fengyou 320 and Fengyou 958 were produced from Pol-CMS hybrid varieties, and Fengyou 737 was self-crossed into $F_{2}$ population.

Table 2 Name and source of 12 rapeseed lines tested in this study

\begin{tabular}{|c|c|c|}
\hline No. & Material name & Source \\
\hline 1 & $20 \mathrm{~A}$ & Pol-CMS sterile line transformed with germplasm from JiangXi \\
\hline 2 & $317 \mathrm{~A}$ & Pol-CMS sterile line bred from reciprocal cross population between 16A and double-low line 317 \\
\hline 3 & $167 \mathrm{~A}$ & Pol-CMS sterile line bred from 17A \\
\hline 4 & $17 \mathrm{~A}$ & $\begin{array}{l}\text { Pol-CMS sterile line bred from Xiang } 5 \mathrm{~A} \text { with pol CMS and double-low line Zhongshuang } 10 \text { by } \\
\text { hybridization and subsequent orientation selection }\end{array}$ \\
\hline 5 & $169 \mathrm{~A}$ & $\begin{array}{l}\text { Pol-CMS sterile line bred from Xiang } 5 \mathrm{~A} \text { with pol CMS and double-low line SC4 by hybridization } \\
\text { and subsequent orientation selection }\end{array}$ \\
\hline 6 & $13 \mathrm{R}$ & Pol-CMS restorer line transformed with Xiangyou 13 \\
\hline 7 & XWR & Pol-CMS restorer line bred from Pol-CMS hybrid in Hubei \\
\hline 8 & Z2R & Pol-CMS restorer line bred from Pol-CMS hybrid Zhongyouza 2 \\
\hline 9 & $320 \mathrm{R}$ & $\begin{array}{l}\text { Pol-CMS restorer line bred from Fengyou } 730 \text { with pol CMS and double-low line } 320 \text { by } \\
\text { emasculation hybridization and subsequent orientation selection }\end{array}$ \\
\hline 10 & $\mathrm{SC} 4$ & Pol-CMS restorer line transformed with germplasms from Sichuan \\
\hline 11 & $\mathrm{SC} 3$ & Pol-CMS restorer line transformed with germplasms from Sichuan \\
\hline 12 & $95-5$ & Pol-CMS restorer line bred from Pol-CMS hybrids in Hubei \\
\hline
\end{tabular}

\subsection{Sampling of test samples}

At the end of September 2018, 12 Pol CMS hybrid parental materials were sown in the experimental field of Hunan Crop Research Institute (the last crop was rice). At the seedling stage (about 7-leaf stage), 5 young leaves of each material were picked out and placed in a $2 \mathrm{~mL}$ centrifuge tube and stored at $-20^{\circ} \mathrm{C}$ for primer screening.

Each sample of Fengyou 737, Fengyou 320 and Fengyou 958 was randomly selected about 1000 seeds and divided into two parts, one for molecular marker purity identification and the other for field planting purity identification.

About $500 \mathrm{~F}_{2}$ population seeds of the tested variety Fengyou 737 were selected and sown in the experimental field of Hunan Crop Research Institute. Each sample identification plot was in 8 rows, with a line length of $2.0 \mathrm{~m}$ and a row spacing of $0.5 \mathrm{~m}$. The seeds were evenly sown and the seedlings were not thinned during the whole growth period.

\subsection{Development of SSR markers linked to $R f p$}

The 1953 bp sequence of $O R F 2$ published by Liu and Zhang (2016) was performed BLAST analysis on the $B$. rapa genome and B. napus genome (http://brassicadb.org/brad/) to identify the physical locations of highly homologous $R f p$ genes related to fertility restoration. Based on the location and its area on both sides of the $5 \mathrm{~K}$, 
$10 \mathrm{~K}, 15 \mathrm{~K}$ for SSR loci, via http://wsmartins.net/websat/ online design SSR primers. Then, the designed SSR primers were used for PCR amplification on genomic DNA of different restorer lines and male sterile lines, and the primers with differences were screened as linkage markers of $R f p$ gene.

\subsection{Verification of cosegregation of fertile segragating populations}

From the $7^{\text {th }}$ to $9^{\text {th }}$ leaf stage, 192 single plants of Fengyou 737 self-pollinating $F_{2}$ population were sequentially selected for tagging, and genomic DNA of single plant was extracted. PCR amplification was performed using the primers screened above, and the amplified products were electrophoresis separated by $3.0 \%$ Agarose gel. Finally, observation, photo taking, and band reading were performed in the gel imaging system. The amplified band types of each individual plant were recorded. In the flowering stage, the fertility performance of 192 plants with tag number was investigated.

\subsection{Identification of purity of hybrid samples by molecular markers}

After the samples of the three varieties were germinated, the genomic DNA of young embryos was extracted according to Chen Ying's CTAB method (Chen, 1993). The genomic DNA of young embryos of Fengyou 737, Fengyou 320 and Fengyou 958 seed production samples were amplified by PCR using primers with different PCR products between sterile and restorer lines. The amplified products were separated by electrophoresis with $3.0 \%$ agarose gel. Finally, the purity of samples was calculated by observing, taking photos and reading bands in the gel imaging system. SSR purity $(\%)=($ number of tested plants-number of maternal band types-number of paternal band types) $\times 100 /$ number of tested plants.

\subsection{Purity identification of hybrid samples in field planting}

The samples to be tested of the three varieties were sown in the test field of Hunan Crop Research Institute. Each sample to be tested was identified in 8 rows with a line length of $2.0 \mathrm{~m}$ and a row spacing of $0.5 \mathrm{~m}$. The seeds were evenly sown, and no seedlings were thinned during the whole growth period. Purity of field identification $(\%)=($ number of tested plants-number of sterile plants-number of heteromorphic plants $) \times 100 /$ number of tested plants.

\section{Authors' contributions}

WTH was the executor of the experimental design and experimental research of this study. WTH, LB and GYM completed the data analysis and drafted the manuscript. LB, GYM, ZYT and LXH participated in the experimental design and analysis of the experimental results. FLY, QL and DLC revised the manuscript and checked the data. LM conceived of the project, directed the design of the study, data analysis, draft and revision. All authors read and approved the final manuscript.

\section{Acknowledgments}

This study was supported by the Science and Technology Innovation Project of Hunan Academy of Agricultural Sciences (2019YC02) and the National Rapeseed Industry Technology System (CARS-12). Thanks to Dr. Liu Zhi for providing the sequence information of target candidate gene for this study.

\section{Reference}

Chen H.Q., Sui C., and Wei J.H., 2009, Summary of strategies for developing ssr primer, Fenzi Zhiwu Yuzhong (Molecular Plant Breeding), 7(4): 845-851

Chen Y., 1993, New methods for extracting plant DNA, Shijie Linye Yanjiu (World Forestry Research), 6(2): 89-91

Fu T.D., 1995, Breeding and utilization of rapeseed hybrid, Hubei Science and Technology Press, Hubei, China, pp. 60-61

He D.H., Lei Z.P., and Xin H.P., 2009, Development progress characteristics and application of functional marker, Xibei Nonglin Keji Daxue Xuebao (Journal of Northwest A and F University (Natural Science Edition)), 37(1): 110-116+121

Hua L.X., Wang W.J., Chen S., Wang C.Y., Zeng L.X., Yang J.Y., Zhu X.Y., and Su J., 2015, Development of specific DNA markers for detecting the rice blast resistance gene alleles Pi2/9/z-t, Zhongguo Shuidao Kexue (Chinese Journal of Rice Science), 29(3): 305-310

Jean M., Brown G..G.., and Landry B.S., 1997, Genetic mapping of nuclear fertility restorer genes for the Polima cytoplasmic male sterility in canola (Brassica napus L.) using DNA markers., Theor. Appl. Genet., 95(3): 321-328

https://doi.org/10.1007/s001220050566

Li H.B., 2016, Mapping and candidate gene analysis of purple leaf gene BnaA.PL1 in Brassica napus L, Dissertation for Ph.D., Huazhong Agricultural Universty, Supervisor: Fu T.D, pp.48-54

Liu F.Q., and Li X., 2007, The application and development of SSR markers in Rape, Zuowu Yanjiu (Crop Research), 21(5): 645-649 
Liu G.Z., and Zhang D.L., 2016, The application of the functional molecular marker in wheat breeding, Shengwu Jishu Tongbao (Biotechnology Bulletin), 32(11): 18-29

Liu P.W., Li Y., He Q.B., and Yang G.S., 2007, Identification of geneticm arkers for the polCM S restorer gene in Brassica napus L., Zhongguo Youliao Zuowu Xuebao (Chinese Journal of Oil Crop Sciences), 29(1): 14-19

Liu Z., 2012, Fine mapping and candidate gene analysis of the nuclear restorer gene for pol CMS in rapeseed (Brassica napus L.), Dissertation for Ph.D., Huazhong Agricultural Universty, Supervisor:Yang G.S, pp.51-56

Liu Z., Liu P.W., Long F.R., Hong D.F., He Q.B., and Yang G.S., 2012, Fine mapping and candidate gene analysis of the nuclear restorer gene Rfp for pol CMS in rapeseed (Brassica napus L.), Theor. Appl. Genet., 125(4): 773-779

https://doi.org/10.1007/s00122-012-1870-2

Mei D.S., Li Y.C., Hu Q., Li Y.D., Xu Y.S., 2006, Identification of seed purity of Brassica napus Zhongyouza no. 8 using SSR markers, Zhongguo Nongxue Tongbao (Chinese Agricultural Science Bulletin), 22(5): 49-52

Natasa F., Li X.Q., Ferrie Alison M.R., DePauw M., Keller W.A., Landry B., and Brown G.G., 2006, Towards positional cloning in Brassica napus: generation and analysis of doubled haploid B. rapa possessing the B. napus pol CMS and Rfp nuclear restorer gene., Plant Mol. Biol., 61(1): 269-281

https://doi.org/10.1007/s11103-006-0008-9

Natasa F., Rachel S., Rachel G., Laetitia M., Martin L., Benoit S.L., and Gregory G..B., 2010, High-resolution mapping of the Brassica napus Rfp restorer locus using Arabidopsis-derived molecular markers., Theor. Appl. Genet., 120(4): 843-851.Song X.H., 2013, Cloning and characterization of resistance gene candidate sequences in two ornamental plants and application of molecular markers for powdery mildew resistance in gerbera, Dissertation for Ph.D., Northwest A\&F University, Supervisor: Ma Q., pp.49-51

https://doi.org/10.1007/s00122-009-1215-y

Tang R., Wu Y.X., Tian Z.P., Zhang M., Wang T.Q., 2007, Hybrid purity identification of Guiyou 519 with yellow seeds by SSR., Guizhou Nongye Kexue (Guuizhou Agricultural Sciences), 35(3): 10-11

Wang H.L., Han J.J., Li M.M., Nan F.B., and Li W.J., 2014, Development and application of functional markers in cereal crops, He Nongxuebao (Journal of Nuclear Agricultural Sciences), 28(11): 1963-1971

Wang P.Q., Zhou Y.F., Xu Y.B., Hu J.B., and Yang L.M., 2016, Development of SSR markers based on multiple sequence alignment and their applications in melon, Nhenan Nongye Daxue Xuebao (Journal of Henan Agricultural University), 50(2): 189-197

Wang X.C., Jiang S.L., Shangguan L.F., Cao Y.F., Qiao Y.S., Zhang Z., and Fang J.G., 2010, Development of EST-derived SSR markers for pear and evaluation of their application in pear genetic diversity analysis, Zhongguo Nongye Kexue (Scientia Agricultura Sinica), 43(24): 5079-5087

Wang Y.X., Zhao R.B., Ding J.Q., Zhang X.C., Chen J.F., Wu K., and Wu J.Y., 2009, Development of novel markers of the resistance gene rscmv1 to maize dwarf mosaic, Henan Nongye Kexue (Journal of Henan Agricultural Sciences), 38(8): 84-87

Yang G.S., Fu T.D., Ma C.Z., and Yang X.N., 1996, Establishment of a random-mating population of 'Polima' CMS restorers in Brassica napus by use of dominant genic male sterility, Plant Breeding, 115(5): 391-394

https://doi.org/10.1111/j.1439-0523.1996.tb00939.X

Yang J.H., Wang S.W., Liu X.Y., Yang J.F., and Zhang M.F., 2008, Development and application of functional markers in higher plants, Zhongguo Nongye Kexue (Scientia Agricultura Sinica), 41(11): 3429-3436

Zeng F.Q., Yi B., Tu J.X., and Fu T.D., 2009, Identification of AFLP and SCAR markers linked to the male fertility restorer gene of pol CMS (Brassica napus L.), Euphytica, 165(2): 363-369

https://doi.org/10.1007/s10681-008-9799-X

Zhan Z.X., Jiang Y.F., Zhu Z.Y., Zhang C.S., Yang Q.Y., Li Q., Hou Z.L., Gong J.F., Chen Y.G., Wu J.S., Fu T.D., Zhou Y.M., Piao Z.Y., and Zhang C.Y., 2015, Development of close linked marker to PbBa8.1 conferring canola resistance to Plasmodiophora brassicae, Zhongguo Youliao Zuowu Xuebao (Chinese Journal of Oil Crop Sciences), 37(6): 766-771

Zhang H.L., Yang Z.H., Hu Z.Z., Zhu J.H., and Zhang AO.C., 2013, Development of genomic microsatellite marker for phytophthora infestans, Nongye Shengwu Jishu Xuebao (Journal of Agricultural Biotechnology), 21(9): 1110-1118

Zhang H.L., Zhu J.H., Hu Z.Z., and Yang Z.H., 2013, Detection and analysis of microsatellites in the phytophthora infestans genome, Beifang Yuan yi (Northern Horticulture), (17): 110-114

Zhao H.X., Li Z.J., Hu S.W., Sun G.L., Chang J.J., and Zhang Z.H., 2010, Identification of cytoplasm types in rapeseed (Brassica napus L.) accessions by a multiplex PCR assay, Theor. Appl. Genet., 121(4): 643-650

https://doi.org/10.1007/s00122-010-1336-3

Zhu Z.G., Dai W.M., Chen X.F., Zhang J.X., and Qiang S., 2015, Development of molecular markers in weedy rice chloroplast based on genomic database, Nanjing Nongye Daxue Xuebao (Journal of Nanjing Agricultural University), 38(2): 240-247 